\title{
Prognostic and predictive value of tumor-infiltrating lymphocytes in breast cancer: a systematic review and meta-analysis
}

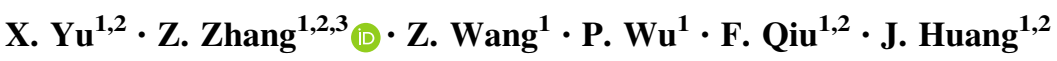

Received: 10 August 2015/Accepted: 17 August 2015/Published online: 12 October 2015

(C) The Author(s) 2015. This article is published with open access at Springerlink.com

\begin{abstract}
Background Breast cancer is the most common invasive cancer to affect women in the world. Studies showed tumor-infiltrating lymphocytes can exhibit both beneficial and harmful effects on the biology and clinical outcome of breast cancer, the conclusion still remains incomplete. Here, we conducted a meta-analysis to evaluate the relationship between tumor-infiltrating lymphocytes and breast cancer.

Methods A comprehensive search strategy was used to search relevant literatures in PubMed and the ISI Web of Science. The correlation among TILs and breast cancer clinicopathological features and prognosis was analyzed by using Review Manager 5.3 and Stata 12.0.
\end{abstract}

$\mathrm{X}$. Yu and $\mathrm{Z}$. Zhang contributed equally to this work.

Electronic supplementary material The online version of this article (doi:10.1007/s12094-015-1391-y) contains supplementary material, which is available to authorized users.

\section{Z. Zhang}

zzg2011@zju.edu.cn

J. Huang

drhuangjian@zju.edu.cn

1 Cancer Institute (Key Laboratory of Cancer Prevention and Intervention, National Ministry of Education, Provincial Key Laboratory of Molecular Biology in Medical Sciences), The Second Affiliated Hospital, Zhejiang University School of Medicine, Hangzhou 310009, China

2 Department of Oncology, Second Affiliated Hospital, Zhejiang University School of Medicine, Hangzhou 310009, China

3 Department of Gynecology, Second Affiliated Hospital, Zhejiang University School of Medicine, Hangzhou 310009, China
Result Seventeen eligible studies consisting of 12,968 participants were included. We found that higher value of tumor-infiltrating lymphocytes had no relationship with breast cancer clinicopathological variables. Interestingly, it was correlated with response to neoadjuvant chemotherapy in majority (pooled RR 2.43, 95 \% CI 1.99-2.97). Moreover, higher value of total tumor-infiltrating lymphocytes (both intraepithelial and stromal) was associated with better prognosis (pooled HR 0.88, $95 \%$ CI 0.83-0.94), whereas some subtypes predicted a worse prognosis.

Conclusion This meta-analysis indicated that high value of total TILs is not associated with breast cancer clinicopathological features, but can predict a favorable outcome for neoadjuvant chemotherapy in majority except for hormone receptor (-) subtype. And higher total TILs (both intraepithelial TILs and stromal TILs) may be the potential better prognostic indicators, while some subtypes like PD$1^{+}$TILs and Foxp3 $3^{+}$TILs show a worse prognosis.

Keywords Tumor-infiltrating lymphocytes - Breast cancer - Clinicopathological features · Prognosis

\section{Introduction}

Breast cancer is one of the most common invasive cancers to affect women health in the world. In 2013, it has accounted for $29 \%$ of all new cancer cases and $14 \%$ of all cancer deaths, becoming the second highest cause of cancer death in women after lung cancer $[1,2]$. Nonetheless, due to the understanding of the breast cancer biology and improvement in early diagnosis and treatment, its mortality has steadily declined. Currently, accumulating evidences have shown that the malignancy and biological features of cancer depend on its genetic abnormalities as well as the interplay between 
cancer cells and microenvironment. Conversely, conventional researches concerned more about the biological features and the prognosis based on the indicators of breast cancer itself, such as histological grade, expression of the hormone receptors (estrogen receptor and progesterone receptor), proliferation marker-Ki67, and amplification status of the HER2 gene et al. [3, 4]. However, recent studies have reported that the tumor microenvironment, comprising adipocytes, tumor-associated fibroblasts, immune cells, extracellular matrix, cytokines and other factors, also plays an important role in tumor formation, growth, invasion and metastasis. Immune cells, like tumor-associated macrophages (TAMs), studies indicated that TAMs generally play a protumoral role, and in the primary tumor, TAMs can stimulate angiogenesis and enhance tumor cell invasion, motility, and intravasation [5, 6]. Clinical evidences indicate the association between high TAMs influx and poor prognosis in breast cancer patients [7, 8]. Also, tumor-infiltrating lymphocytes (TILs) have distinct roles in modulation of the tumor niche, favoring or inhibiting carcinogenesis and cancer progression. Moreover, some subtypes of lymphocytes secrete IL- 6 and IL8 , which in turn activate PI3K/AKT, NF- $\kappa B$, STAT3 signaling, and generate a positive feedback loop between the tumor cells and immune microenvironment $[9,10]$.

Limited data showed that total TILs were associated with a better prognosis $[11,12]$. In breast cancer, TILs existence before chemotherapy is a good phenomenon, which prompts the therapeutic effect of neoadjuvant therapy $[13,14]$. But the type, density, and location of TILs in breast cancer exhibit different values for assessing disease prognosis and progression. The majority of TILs are prominent $\mathrm{CD}^{+} \mathrm{T}$ cells, which are the major effector cell type, and have been linked to a better prognosis [15]. However, Foxp $3^{+} \mathrm{T}$ cells or PD- ${ }^{+} \mathrm{T}$ cells infiltration mediates tumor immune escape and reminds a worse prognosis [16]. Thus, subtypes of TILs can exert both inhibitory and stimulatory effects on breast cancer and the prognostic value of TILs remains complex and controversial. To address this controversy, we conducted a metaanalysis aimed to evaluate the total or subtype of TILs as a potential prognostic marker for breast cancer and to determine the relationship between TILs and several clinicopathological features.

\section{Materials and methods}

\section{Literature search}

This systematic review and meta-analysis were conducted according to the Preferred Reporting Items for Systematic Reviews and Meta-Analyses (PRISMA) statement [17]. A systematic literature search for the following tags: "tumor- infiltrating lymphocytes and breast cancer" or "tumor-associated lymphocytes and breast cancer"-related papers in the electronic databases PubMed and the Web of Science from January 1990 to July 2014 was conducted independently by two investigators. The citation lists associated with the studies, including review articles that were retrieved in the search, were used to identify additional relevant publications. The title and abstract of each study identified in the search were scanned to exclude any clearly irrelevant reports.

\section{Selection criteria}

The studies included in this meta-analysis were either randomized controlled studies (RCTs) or observational studies (case-control or cohort) that evaluated the association between TILs and breast cancer. The criteria for inclusion were as follows: (a) articles evaluating the relationship between TILs and parameters such as clinicopathological features and prognostic factors of breast cancer; (b) articles containing sufficient published data to determine an estimate of relative risk (RR) or hazard ratio (HR) and a $95 \%$ confidence interval $(95 \% \mathrm{CI})$; and (c) full-text, original research articles published in English.

The following studies were excluded: (1) overlapping articles or duplicate data; (2) non-English languages; (3) the types of reviews, comments or letters; (4) articles published in books; and (5) lacking information.

\section{Data extraction}

Data from eligible studies were independently extracted in a standardized manner by the two investigators. Disagreements in data extraction were resolved by consensus and by referring back to the original article. The following data were obtained from each article: first author's last name; year of publication; country of the population studied; number of participants; duration of follow-up; the choice of cutoff scores for the definition of positive staining or staining intensity; $\mathrm{T}$ category (T0-1, T2-4); $\mathrm{N}$ category; HER-2, hormone receptor status; and most importantly, pathological complete response (pCR) rate, the 5-year overall survival (OS) and disease-free survival (DFS) rates.

The cutoff value for the TILs varied among studies, we defined higher expression of TILs value according to the original articles. And high TILs were defined as higher value of total TILs on hematoxylin and eosin (H\&E)stained sections. The $\mathrm{T}$ category was determined according to the American Joint Committee on Cancer (AJCC) cancer staging manual (one group T0-1, other group T2-4). We defined hormone receptor positive either ER $\geq 1 \%$ or PR $\geq 1 \%$ under immunohistochemistry (IHC), and classified HER-2 positive if HER-2 gene amplification using in suit 
hybridization(ISH) or scored as $3+$ by IHC method. To avoid bias from studies contributing very long-term followup data compared with other studies, both OS and DFS rates were standardized to include 5 years of follow-up in all studies. For the articles that did not provide 5-year OS and DFS rates directly, Kaplan-Meier curves were evaluated using GetData Graph Digitizer 2.24 (http:// getdatagraph-digitizer.com).

\section{Assessment of study quality}

The qualities of 17 eligible studies included in our metaanalysis were assessed according to the Newcastle-Ottawa scale (NOS). The NOS contains eight items, which are categorized into the three dimensions of selection, comparability, and outcome (cohort studies) or exposure (casecontrol studies). The quality scores in NOS ranged from 0 (lowest) to 9 (highest) and studies with scores 6 or more were rated as high quality. All included studies obtained scores of 6 or more in the methodological assessment, indicating that they were of high quality (Table 1).

\section{Statistical analysis}

This meta-analysis calculated the pooled RR or HR with its corresponding $95 \% \mathrm{CI}$ to assess the association of TILs with breast cancer using RevMan 5.3. Study heterogeneity was measured using the $Q$ test and $I^{2}$ test. Fixed-effects models (Mantel-Haenszel, $P>0.1$ and $I^{2}<50 \%$ ) assume that the differences between the results of various studies are due to chance. Random-effects models (DerSimonian and Laird, $P \leq 0.1$ or $I^{2} \geq 50 \%$ ) assume that the results can genuinely differ between studies. In the absence of heterogeneity, both fixed- and random-effects models provide similar results. When heterogeneity is present, the random-effects model is considered to be more appropriate than a fixed-effects model, resulting in wider intervals and a more conservative estimate of effect. The publication bias on the reported outcomes was assessed with the construction of contour-enhanced funnel plots and Egger's tests; this analysis was performed by STATA version 12.0.

\section{Results}

\section{Search results and characteristics of eligible studies}

The detailed search steps are described in Fig. 1. Initially, 1833 potential articles were retrieved utilizing the search strategy described above. After titles and abstracts were reviewed, 1679 articles were excluded. Thus, 154 full-text papers was viewed, of these papers, another 135 were excluded because they did not provide data between TILs and clinicopathological features or specify whether disease-free survival (DFS)/overall survival (OS) rate was investigated. Finally, a total of 17 studies were included for the meta-analysis.

All features of the eligible studies in systematic review and meta-analysis are summarized (Table 1). These observational retrospective studies evaluated the level of TILs and clinicopathological features or prognostic parameters for breast cancer, consisting of approximately 12,968 participants with a median of 711 (from 68 to 3403) per study and with a median follow-up of 73 months (range 39-180). Among them, five were from the Europe, four from America, seven from Asia, and one from Africa, which covered the most areas around the world.

\section{Higher value of total TILs was not associated with breast cancer clinicopathological features, but some subtypes may have}

High TILs value was not associated with certain clinical parameters of breast cancer, such as grade category (G1-2 vs. G3): (pooled RR 0.93, $95 \%$ CI 0.77-1.13); hormone receptor status (+ vs. - ): (pooled RR $0.48,95 \% \mathrm{CI}$ 0.07-3.32); or HER-2 status (+ vs. - ): (pooled RR 0.83, $95 \%$ CI 0.61-1.12). So, we found that total TILs were not associated with tumor grade, hormone receptor or HER-2 status. Unfortunately, we had not analyzed the relationship of tumor size, lymph node status and Ki-67, due to the limited quantity of literatures (Supplementary Figure 1).

It is already known that TILs in breast cancer have several subtypes, such as $\mathrm{CD} 8^{+} \mathrm{T}$ cell, $\mathrm{PD}-1^{+} \mathrm{T}$ cell and Foxp $3+\mathrm{T}$ cell. Through the analysis, we found that PD- ${ }^{+}$ TILs were related to high tumor grade (G1-2 vs. G3) (pooled RR 0.63, $95 \%$ CI 0.54-0.73), big tumor size (T1 vs. T2-4) (pooled RR 0.72, $95 \%$ CI 0.62-0.82), positive lymph node (+ vs. -) (pooled RR 1.76, $95 \%$ CI 1.50-2.07), negative hormone receptor (+ vs. - ) (pooled RR 0.75, $95 \%$ CI 0.66-0.84) and HER-2 status (+ vs. -) (pooled RR 1.53, $95 \%$ CI 1.08-2.16). Both Foxp3 ${ }^{+}$TILs and $\mathrm{CD} 8^{+}$TILs also had some relationships with breast cancer clinicopathological parameters (Table 2).

\section{Higher value of total TILs predicted a better response to neoadjuvant chemotherapy in most breast cancers, except hormone receptor negative ones}

We defined the pathological complete response ( $\mathrm{pCR}$ ), which meant no residual invasive cancer cells in surgical specimens of primary tumor and axillary lymph node. Six studies containing 1970 patients were selected. The results showed overexpression of total TILs predicted a higher pCR rate (pooled RR 2.43, $95 \%$ CI 1.99-2.97). Besides, high-TILs were also associated with elevated $\mathrm{pCR}$ rate for hormone receptor $(+)$, 


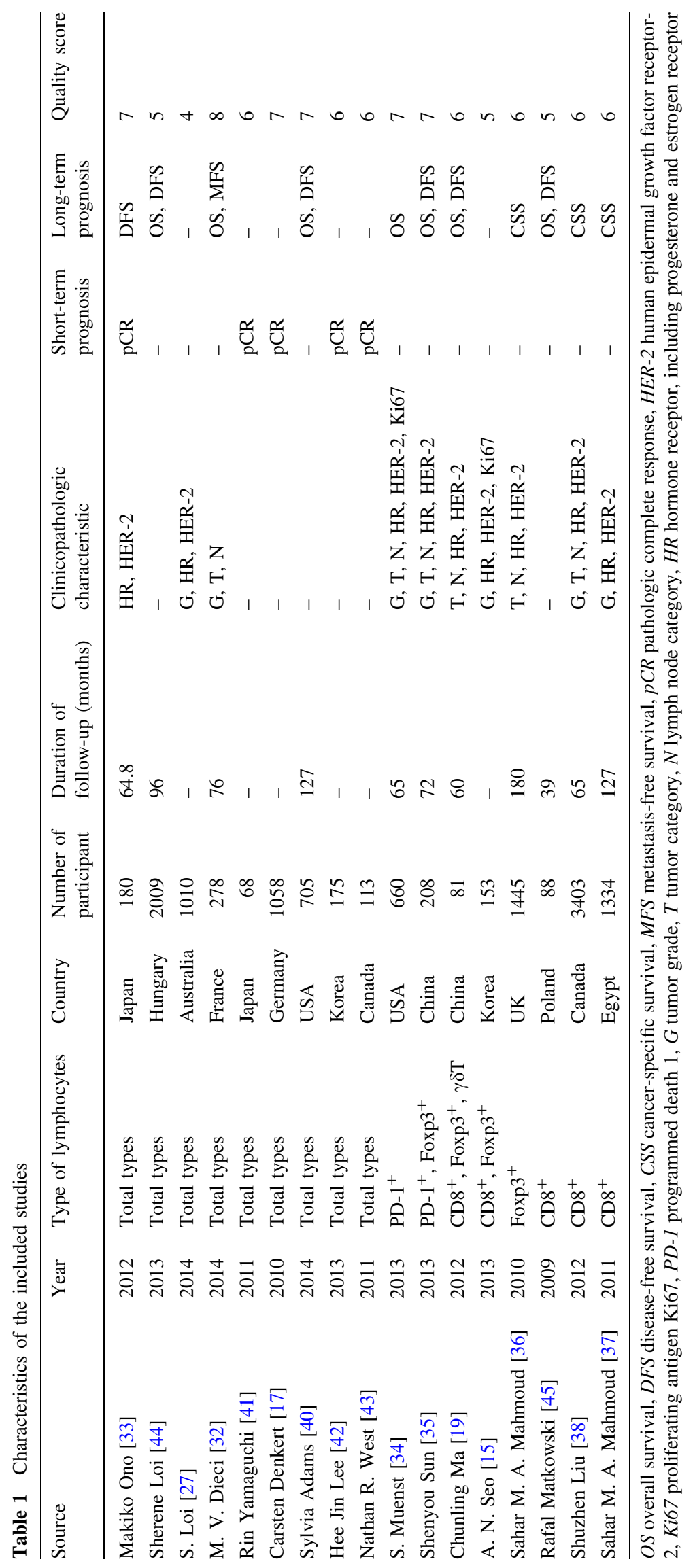


Fig. 1 Flowchart of the selection of studies for inclusion in the meta-analysis

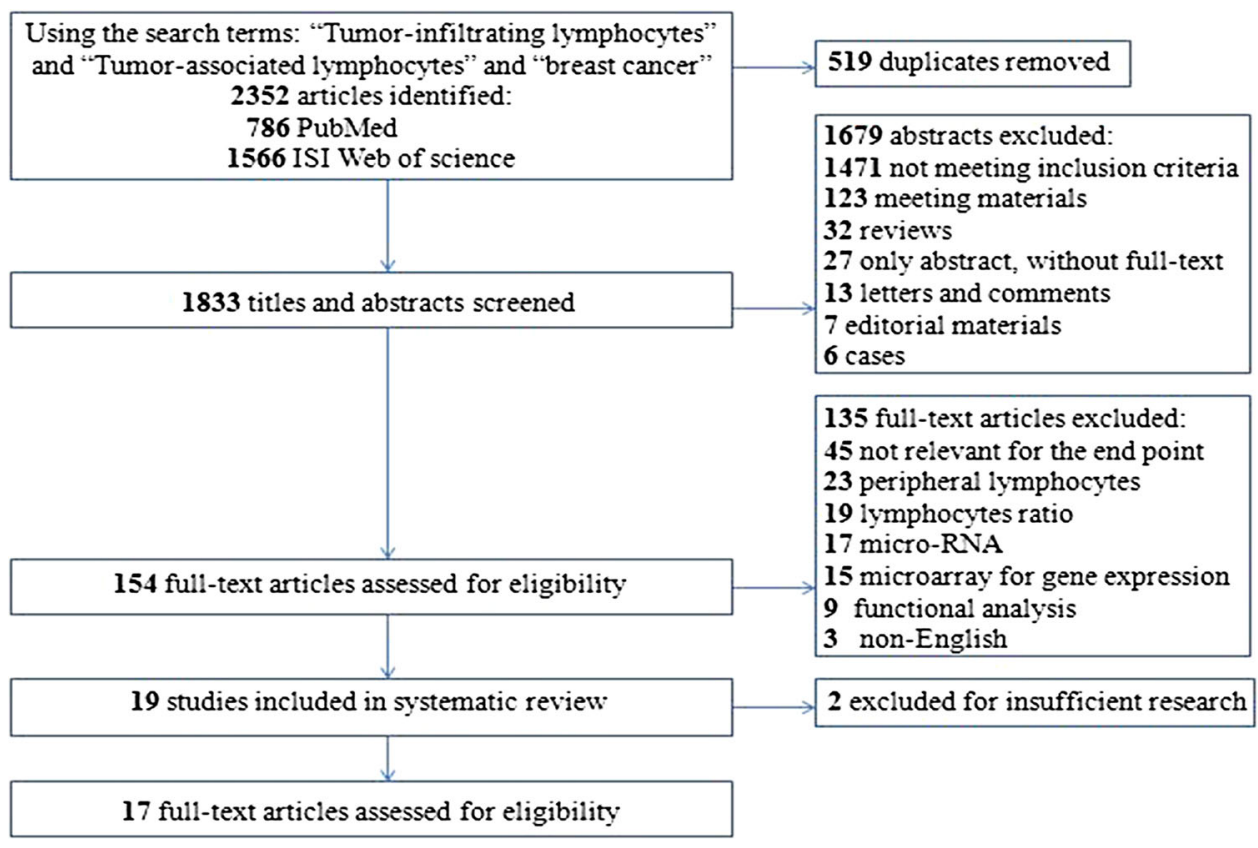

HER-2 (+), and HER-2 (-) breast cancer, respectively (pooled RR were 2.24, 1.92, and 2.68, respectively) (Fig. 2).

\section{Higher value of total TILs was significantly associated with better prognosis, but several subtypes revealed a worse result}

Three studies (including a total of 2992 patients) that demonstrated the association of total TILs and the longterm survival were obtained from the published data. Metaanalysis of the included papers reporting DFS and metastasis-free survival in total TILs revealed a pooled HR of 0.88 , with a $95 \%$ CI of $0.83-0.98$, which is statistically significant $(P<0.0001)$. Total TILs also indicate a long overall survival, but without statistically significant $(P=0.08)$. We also evaluated the prognostic utility of TILs within intraepithelial (iTILs) and stromal compartments (sTILs). The pooled data suggest both iTILs and sTILs were associated with better DFS and cancer-specific survival, estimated HRs in iTILs and sTILs being 0.90 (95\% CI $0.83-0.98)$ and 0.85 (95\% CI $0.76-0.94)$, whereas they were not significantly correlated with OS (HR in iTILs $0.90,95 \%$ CI $0.76-1.06$ and HR in sTILs 0.91, $95 \%$ CI 0.77-1.08) (Fig. 3).

In a subgroup analysis, both PD- ${ }^{+}$TILs (polled HR 2.92, $95 \%$ CI 1.81-4.72) and Foxp3 $3^{+}$TILs (polled HR $3.86,95 \%$ CI 1.62-9.22) predicted poor overall survival. But there exists no significance for disease-free survival and cancer-specific survival. Breast cancer with high level of $\mathrm{CD}^{+}$TILs showed a favorable disease-free survival (pooled HR 0.52, 95 \% CI 0.30-0.69) (Fig. 4).
Wen-Chung Che's research illustrated that interleukin17-producing TILs had a survival influence on breast cancer, this study contained 207 breast cancer patients, the result indicated that IL-17-producing TILs were associated with high grade, hormone receptor (-) subtype, and a poorer survival (disease-free survival 64.0 vs. $87.3 \%$; HR 2.68; $95 \%$ CI 1.37-5.27; $P<0.01$ ) [18]. In addition, there was another small group called $\gamma \delta \mathrm{T}$ TILs in breast cancer. These $\gamma \delta \mathrm{T}$ cells were very common in breast cancer microenvironment, and predicted a better survival (recurrence-free survival: HR $41.6995 \%$ CI $5.4-321.96 ; P=4.79 \times 10^{-8}$. Overall survival: HR $44.73 \quad 95 \% \mathrm{CI} \quad 5.79-345.22, \quad P=$ $1.51 \times 10^{-8}$ ) [19].

\section{Publication bias}

Egger's tests indicated that there was no evidence of significant publication bias after assessing the funnel plot for the studies included in our meta-analysis (Supplementary Figure 2).

\section{Discussion}

The information about the prognostic and predictive value of TILs in breast cancer is still limited. To our best knowledge, the present meta-analysis is the first study to systematically evaluate the association among TILs with clinical-pathological features and prognostic factors in breast cancer. 


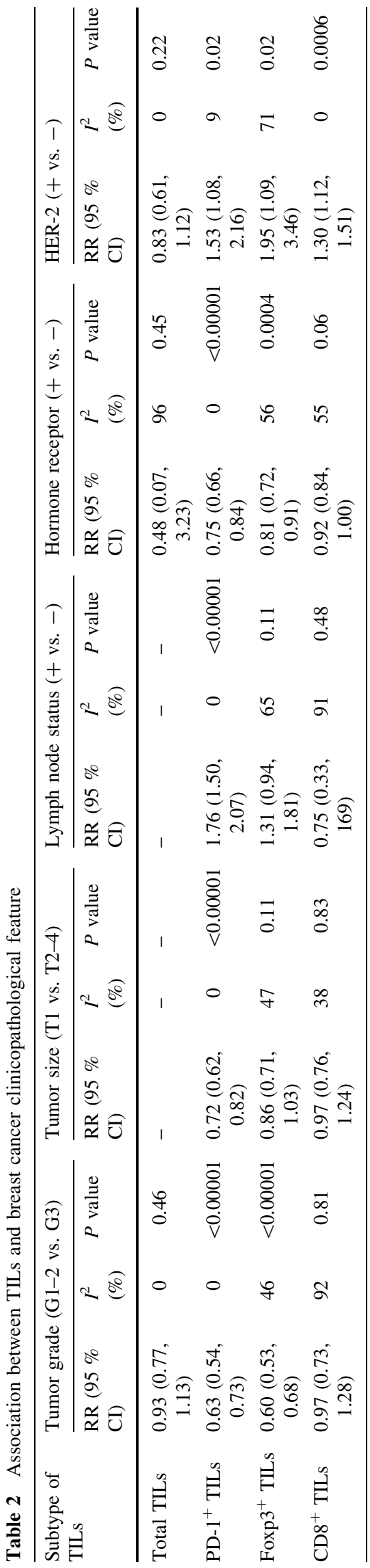

\section{Correlation of TILs with clinicopathological parameters}

Total TILs in breast cancer are not strongly associated with clinicopathological characteristics, the previous studies showed that breast cancer with high differentiation, hormone receptor (-) and HER-2 (+) would have a higher level of TILs [13]. But our study did not achieve the similar result; this might be related to very small available raw data and lack of large size of sample research. But PD$1^{+}$and Foxp $3^{+}$subtype TILs highly expressed in hormone receptor $(-)$, HER-2(+) breast cancer, which have a high risk of recurrence and metastasis. However, $\mathrm{CD} 8^{+} \mathrm{TILs}$ as the core of the local immune cells did not have relationship with clinic pathological traits of breast cancer.

\section{Relationship between TILs and neoadjuvant chemotherapy response}

Recently, the relationship between TILs and the response of breast cancer with neoadjuvant chemotherapy have attracted much attention. Most researches deem that rich of TILs can improve the sensitivity and effect of neoadjuvant chemotherapy. The reasons mainly include (Fig. 5): first, influencing the factors about tumor immunosurveillance. Chemotherapeutic drugs were selected for their direct cytotoxic effects against highly proliferative tumor cells, releasing tumor antigen, ATP and purinergic receptor. Tumor cells expressed HLA-I had higher $\mathrm{CD} 8^{+} \mathrm{T}$ cell infiltration [20]. Besides, ATP-dependent pathway whereby the intratumoral accumulation of granulocytemonocyte progenitors (GMPs) and inflammatory monocytes facilitates the local differentiation of inflammatory DCs and the activation of $\mathrm{T}$ cells against cancer [21]. Chemotherapy promoting chemokine expression in tumor microenvironment affects leukocyte migration, such as CCL2/CCR2 pathway reboots antigen-specific $\mathrm{T}$ cell responses [22]. Otherwise, chemotherapy can induce IL-2 and IFN $\gamma$ secretion to trigger immunogenic cell death, and increase the permeability of tumor cells to granzyme B, thereby rendering them to be susceptible to CTL-mediated lysis even if they do not express the antigen recognized by CTLs [9]. Second, influencing the factors about tumor immunosuppression. Regulatory $\mathrm{T}$ lymphocytes (Tregs) and myeloid-derived suppressor cells (MDSCs) are major components of these inhibitory cellular networks [23]. Compared with other immune cell, Tregs have a higher proliferation rate and can be directly "killed" by chemotherapy. Drugs, like cyclophosphamide, can impair Treg-suppressive function by downregulating Foxp3 and glucocorticoid-induced TNFR-related protein. The selective induction of Tregs apoptosis by paclitaxel was attributed to the upregulation of the cell death receptor Fas 


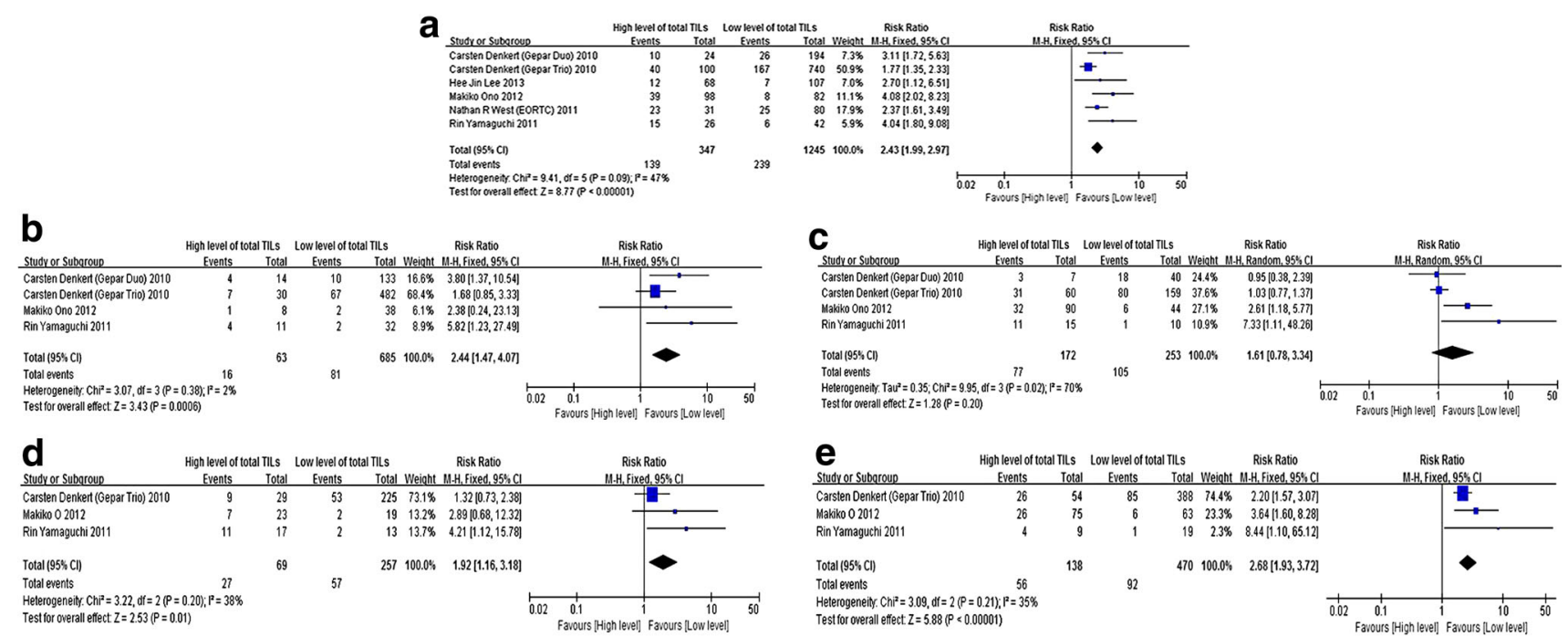

Fig. 2 The forest plot of RRs was assessed for association between TILs and breast cancer short-term outcome (neoadjuvant chemotherapy pCR rate). a TILs and breast cancer, b TILs and hormone

receptor $(+)$ breast cancer, c TILs and hormone receptor $(-)$ breast cancer, d TILs and HER-2 (+) breast cancer, e TILs and HER-2 (-) breast cancer
Fig. 3 The forest plot of HRs was assessed for association between total TILs and breast cancer long-term prognosis. a Total TILs and disease-free survival/metastasis-free survival, b total TILs and overall survival

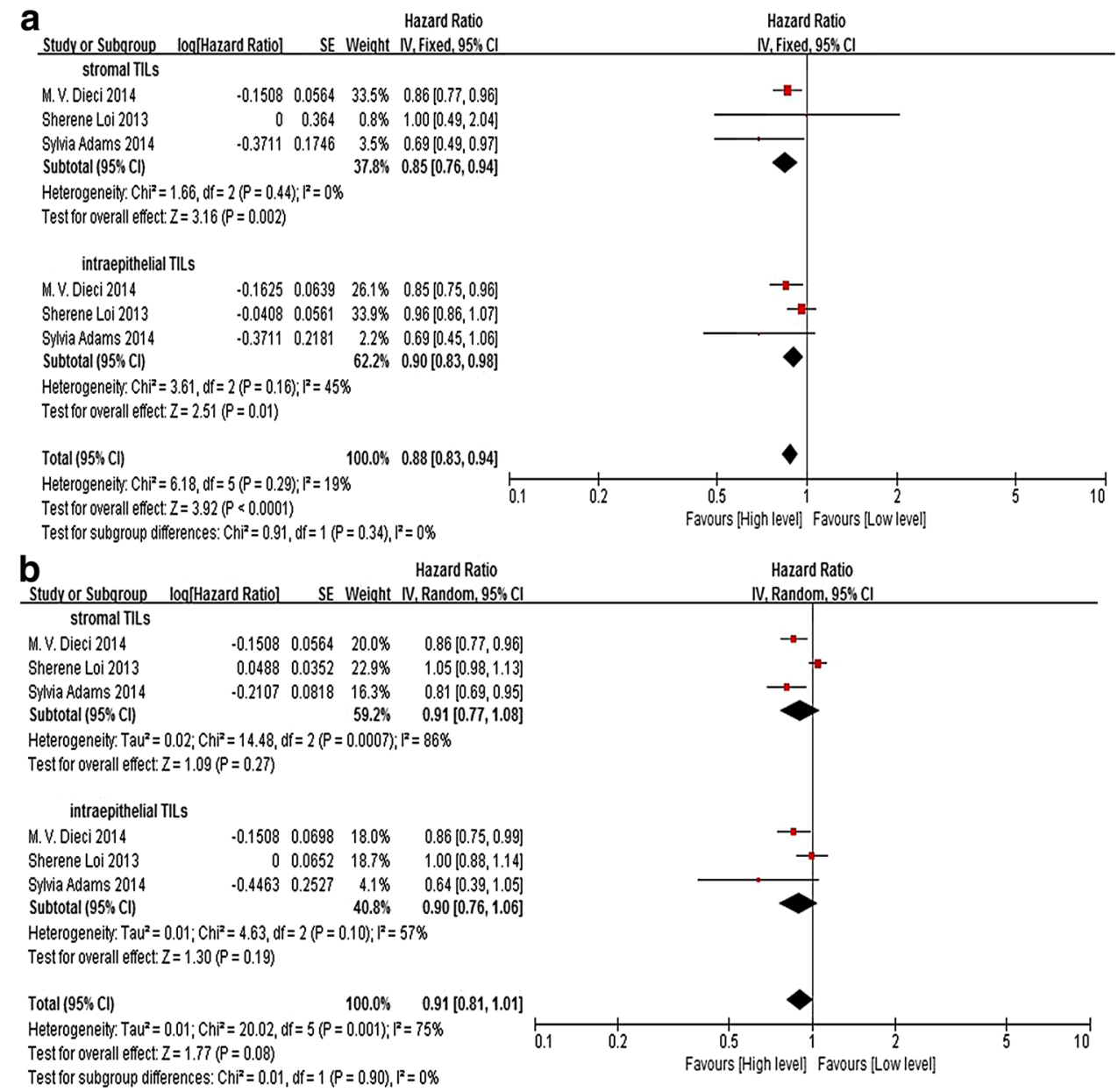




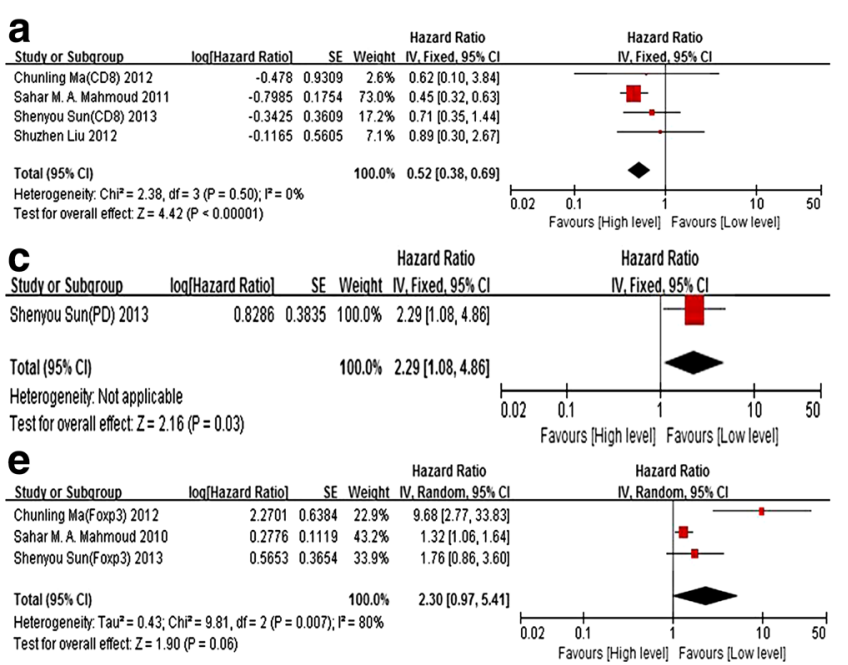

Fig. 4 The forest plot of HRs was assessed for association between subtypes of TILs and breast cancer long-time prognosis. a CD8 ${ }^{+}$TILs and disease-free survival/cancer-specific survival, $\mathbf{b} \mathrm{CD} 8^{+}$TILs and

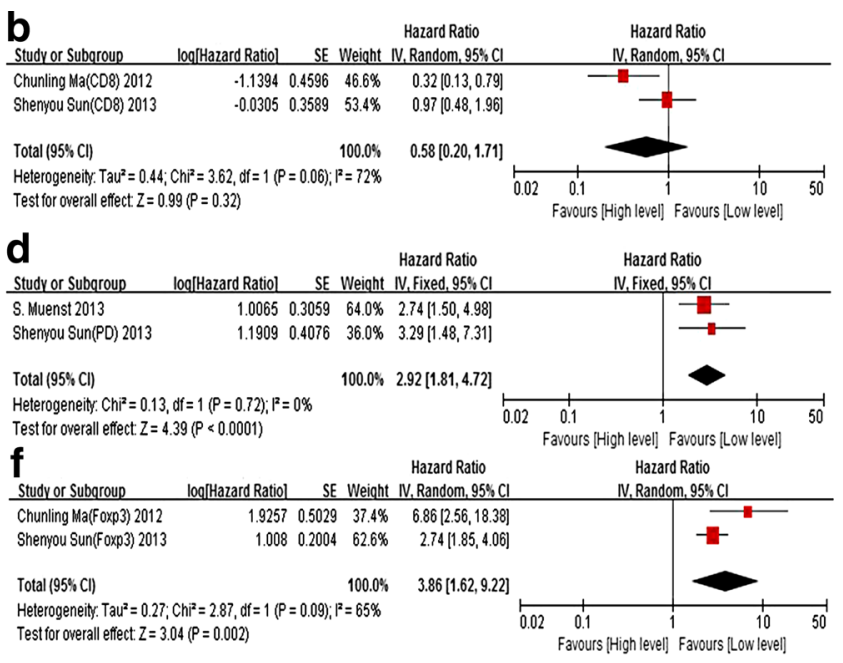

overall survival, c PD-1 ${ }^{+}$TILs and disease-free survival, d PD- $1^{+}$ TILs and overall survival, e Foxp $3^{+}$TILs and disease-free survival/cancer-specific survival, f Foxp $3^{+}$TILs and overall survival
Fig. 5 A positive feedback loop between TILs and breast cancer chemotherapy

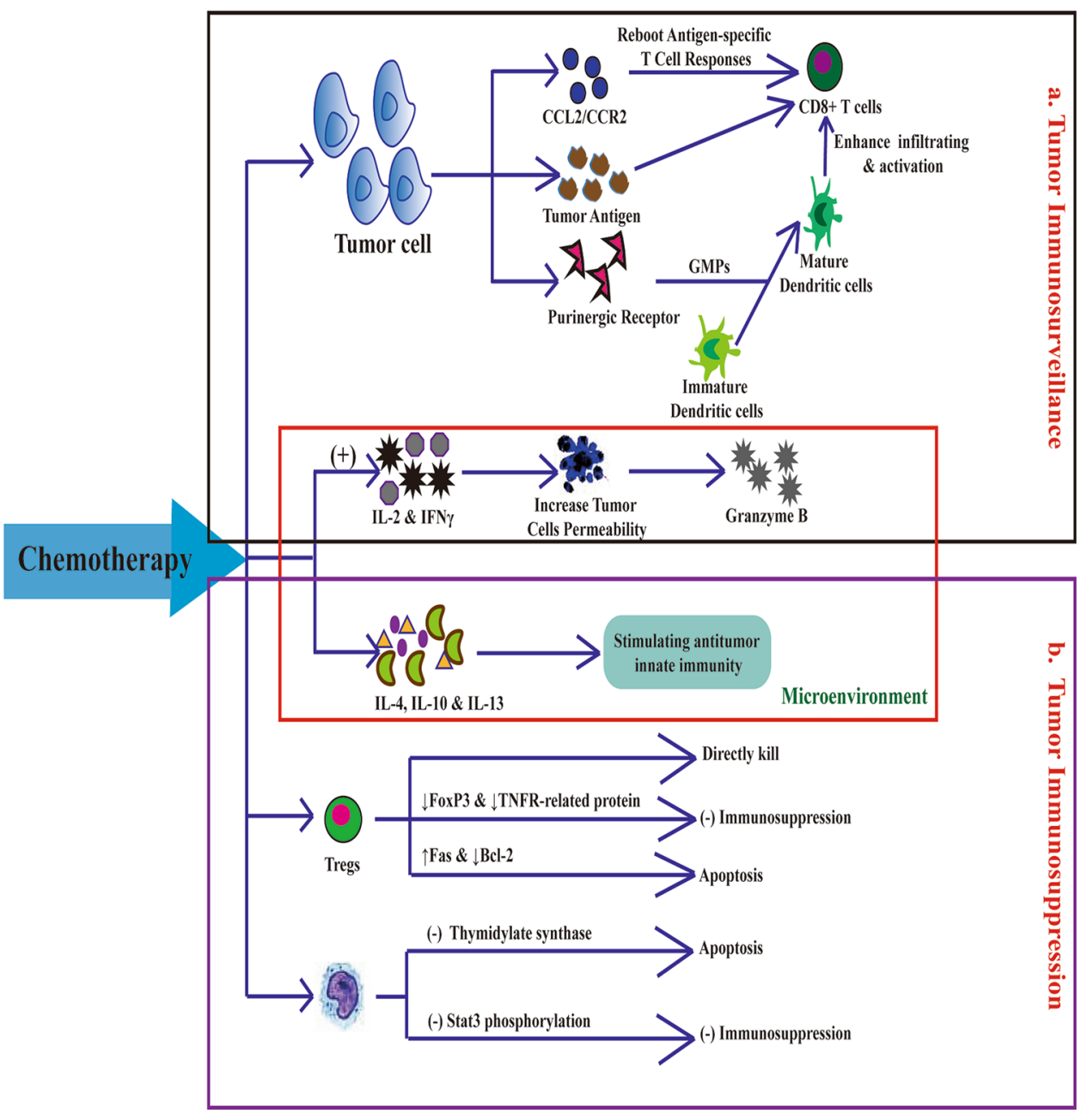


and downregulation of the antiapoptotic molecule Bcl-2 on Tregs [24]. The antimetabolite like 5-fluorouracil at low doses had also been shown to induce MDSCs apoptosis, this selective effect was explained by a lower expression of thymidylate synthase by MDSCs [25]. Docetaxel and paclitaxel were shown to impair MDSCs suppressive function, predominantly by blocking Stat3 phosphorylation and by promoting MDSC differentiation into M1 macrophages or dendritic cells (DCs). Chemotherapy can also inhibit immunosuppressive cytokines (including IL-4, IL10 and IL-13) while stimulating antitumor innate immunity [20].

Our study reflected that high-TILs showed a better treatment response of neoadjuvant chemotherapy, but under subtype analysis, we found in hormone receptor $(-)$ breast cancer did not get the same result. This result differed from the Clinical Trial PrECOG0105 [26]. In 2014, American Society of Clinical Oncology (ASCO), Shaveta Vinayak reported that TILs were predictive of response to platinum-based neoadjuvant therapy and were significantly associated with triple-negative breast cancer (TNBC), the causes and possible reasons for this difference may be the chemotherapy agents, eligible studies in our analysis almost used the anthracycline-based chemotherapy.

In 2013, San Antonio Breast Cancer Symposium (SABCS), Sherene Loi reported that TILs were associated with higher pCR rates after neoadjuvant trastuzumab and chemotherapy in early-stage HER2-positive breast cancer [27]. This research gave us a new message that tumor targeted therapy and TILs had a complex relationship, more studies were needed to explore TILs dynamic change with trastuzumab treatment, and the mechanism [28].

Breast cancer is a kind of malignant tumor with high heterogeneity, TILs as a marker for treatment need adequate consideration about the molecular subtypes of cancer and chemotherapy agents. Certain types of breast cancer with appropriate level of chemotherapy enhance local immune reaction and activate cytotoxic lymphocytes. Immune reaction and conventional antineoplastic agents play a double effect on breast cancer and finally improve the therapeutic outcome.

\section{Impact of TILs on long-term prognosis}

Previous studies had shown that higher expression of total TILs suggested a better prognosis of breast cancer, our study also got the similar results. But we found that the value for prognostic implication was not affected by TILs location, both iTILs and sTILs contributed to a favorable survival.

It is worth noting that different TILs subtypes have different results. High level of PD- $1^{+}$TILs or Foxp $3^{+}$TILs predicts a poor prognosis. PD- $1^{+}$TILs or Foxp $3^{+}$TILs can suppress antitumor immune response and lead to escape immune clearance, so the more the PD- $1^{+}$TILs or Foxp $3^{+}$ TILs were, the worse the prognosis of patients was [29, 30]. On the contrary, $\mathrm{CD}^{+}$TILs indicated a good prognosis [31]. These results therefore reflect that lymphocytes in tumor microenvironment can affect the balance of immune response and tolerance, leading to different outcome.

Most studies indicated the association between TILs in pre-treatment and long-term prognosis of breast cancer, but what was it about TILs in residual lesions after neoadjuvant therapy? M. V. Dieci proved that the higher concentration of residual lesions TILs after neoadjuvant therapy predicted a better prognosis, and this is not affected by chemotherapy cycle times [32]. So, TILs dynamics variation may be a good prognostic marker in breast cancer microenvironment.

However, there still exist some limitations in the present meta-analysis. First of all, the literatures in the metaanalysis were based on observational studies, the correctness of the result depended on the accuracy of the original literature research, we therefore formulated the strict inclusion and exclusion standard. Moreover, this study only included the published literature in English and limited quantity literatures, there might exist language bias and study heterogeneity.

In conclusion, our study shows a significant correlation between TILs and clinical traits in breast cancer patients. Higher value of total TILs not only predicts neoadjuvant chemotherapy response, but also implies a better prognosis, whereas some subtypes of TILs, like PD- $1^{+}$TILs and Foxp $3^{+}$TILs, are not amity with breast cancer and predict an unfavorable outcome. But for hormone receptor (-) breast cancer, neoadjuvant chemotherapy agent may affect the TILs infiltrating, and lead to a different therapeutic effect. So TILs should be monitored in breast cancer patients for rational stratification and adjusting the treatment strategy, further meticulous and deep researches about TILs and breast cancer are also needed.

Acknowledgments We would like to thank Dr Yihua Wu, at Zhejiang University School of Public Health, for study design and critical review.

\section{Compliance with ethical standards}

Conflict of interest The authors declare no conflict of interest.

Open Access This article is distributed under the terms of the Creative Commons Attribution 4.0 International License (http:// creativecommons.org/licenses/by/4.0/), which permits unrestricted use, distribution, and reproduction in any medium, provided you give appropriate credit to the original author(s) and the source, provide a link to the Creative Commons license, and indicate if changes were made. 


\section{References}

1. Siegel R, Ma J, Zou Z, Jemal A. Cancer statistics, 2014. CA Cancer J Clin. 2014;64(1):9-29. doi:10.3322/caac.21208.

2. Fan L, Strasser-Weippl K, Li J-J, St Louis J, Finkelstein DM, Yu K-D, et al Breast cancer in China. Lancet Oncol. 2014;15(7):e279-89. doi:10.1016/s14702045(13)70567-9.

3. Mouawad R, Spano JP, Khayat D. Lymphocyte infiltration in breast cancer: a key prognostic factor that should not be ignored. J Clin Oncol 2011;29(15):1935-6. doi:10.1200/jco.2011.35.4845.

4. Hanahan D, Weinberg Robert A. Hallmarks of cancer: the next generation. Cell. 2011;144(5):646-74. doi:10.1016/j.cell.2011.02.013.

5. Noy R, Pollard JW. Tumor-associated macrophages: from mechanisms to therapy. Immunity. 2014;41(1):49-61. doi:10.1016/j.immuni.2014.06.010.

6. Raggi C, Mousa HS, Correnti M, Sica A, Invernizzi P. Cancer stem cells and tumor-associated macrophages: a roadmap for multitargeting strategies. Oncogene. 2015. doi:10.1038/onc.2015.132.

7. Hu W, Li X, Zhang C, Yang Y, Jiang J, Wu C. Tumor-associated macrophages in cancers. Clin Transl Oncol. 2015. doi:10.1007/s12094-015-1373-0.

8. Tang X. Tumor-associated macrophages as potential diagnostic and prognostic biomarkers in breast cancer. Cancer Lett. 2013;332(1):3-10. doi:10.1016/j. canlet.2013.01.024.

9. Korkaya H, Liu S, Wicha MS. Breast cancer stem cells, cytokine networks, and the tumor microenvironment. J Clin Invest. 2011;121(10):3804-9. doi:10.1172/ jci57099.

10. Perdiguero EG, Geissmann F. Identifying the infiltrators. Science. 2014;344(6186):801-2. doi:10.1126/science.1255117.

11. Gajewski TF, Schreiber H, Fu Y-X. Innate and adaptive immune cells in the tumor microenvironment. Nat Immunol. 2013;14(10):1014-22. doi:10.1038/ni. 2703

12. Cruz-Merino L, Barco-Sanchez A, Henao Carrasco F, Nogales Fernandez E, Vallejo Benitez A, Brugal Molina J, et al. New insights into the role of the immune microenvironment in breast carcinoma. Clin Dev Immunol. 2013;2013:785317. doi:10.1155/2013/785317.

13. Denkert C, Loibl S, Noske A, Roller M, Muller BM, Komor M, et al. Tumorassociated lymphocytes as an independent predictor of response to neoadjuvant chemotherapy in breast cancer. J Clin Oncol. 2010;28(1):105-13. doi:10.1200/ JCO.2009.23.7370.

14. Martinet L, Garrido I, Filleron T, Le Guellec S, Bellard E, Fournie JJ, et al. Human solid tumors contain high endothelial venules: association with T- and B-lymphocyte infiltration and favorable prognosis in breast cancer. Cancer Res. 2011;71(17):5678-87. doi:10.1158/0008-5472.can-11-0431.

15. Seo AN, Lee HJ, Kim EJ, Kim HJ, Jang MH, Lee HE, et al. Tumour-infiltrating $\mathrm{CD}^{+}$lymphocytes as an independent predictive factor for pathological complete response to primary systemic therapy in breast cancer. $\mathrm{Br} \mathrm{J}$ Cancer. 2013;109(10):2705-13. doi:10.1038/bjc.2013.634

16. Takenaka M, Seki N, Toh U, Hattori S, Kawahara A, Yamaguchi T, et al. FOXP3 expression in tumor cells and tumor-infiltrating lymphocytes is associated with breast cancer prognosis. Mol Clin Oncol. 2013;1(4):625-32. doi:10. 3892/mco.2013.107.

17. Liberati AAD, Tetzlaff J, Mulrow C, Gøtzsche PC, Ioannidis JP, Clarke M, et al. The PRISMA statement for reporting systematic reviews and meta-analyses of studies that evaluate health care interventions: explanation and elaboration. PLoS Med. 2009;6(7):e1000100. doi:10.1371/journal.pmed.1000100.

18. Chen WC, Lai YH, Chen HY, Guo HR, Su IJ, Chen HH. Interleukin-17-producing cell infiltration in the breast cancer tumour microenvironment is a poor prognostic factor. Histopathology. 2013;63(2):225-33. doi:10.1111/his.12156.

19. Ma C, Zhang Q, Ye J, Wang F, Zhang Y, Wevers E, et al. Tumor-infiltrating gamma delta $\mathrm{T}$ lymphocytes predict clinical outcome in human breast cancer. J Immunol. 2012;189(10):5029-36. doi:10.4049/jimmunol.1201892.

20. Ma Y, Adjemian S, Mattarollo Stephen R, Yamazaki T, Aymeric L, Yang H, et al. Anticancer chemotherapy-induced intratumoral recruitment and differentiation of antigen-presenting cells. Immunity. 2013;38(4):729-41. doi:10.1016/j. immuni.2013.03.003.

21. Zitvogel L, Apetoh L, Ghiringhelli F, Kroemer G. Immunological aspects of cancer chemotherapy. Nat Rev Immunol. 2008;8(1):59-73. doi:10.1038/ nri2216.

22. Yuting Ma SRM, Adjemian Sandy, Yang Heng, Aymeric Laetitia, Dalil Hannani J, Catani Paulo Portela, et al. CCL2/CCR2-dependent recruitment of functional antigen-presenting cells into tumors upon chemotherapy. Cancer Res. 2014;74(2):436-45. doi:10.1158/0008-5472.CAN-13-1265.

23. Alizadeh D, Larmonier $\mathrm{N}$. Chemotherapeutic targeting of cancer-induced immunosuppressive cells. Cancer Res. 2014;74(10):2663-8. doi:10.1158/00085472.can-14-0301.

24. Ye J, Ma C, Wang F, Hsueh EC, Toth K, Huang Y, et al. Specific recruitment of regulatory $\mathrm{T}$ cells in human breast cancer. Cancer Res. 2013;73(20):6137-48. doi:10.1158/0008-5472.can-13-0348.
25. Tongu M, Harashima N, Monma H, Inao T, Yamada T, Kawauchi H, et al Metronomic chemotherapy with low-dose cyclophosphamide plus gemcitabine can induce anti-tumor T cell immunity in vivo. Cancer Immunol Immunother. 2012;62(2):383-91. doi:10.1007/s00262-012-1343-0.

26. Oncology OJotASoC. Abstracts American Society of Clinical Oncology 50th Annual Meeting. J Clin Oncol. 2014;32(15S):48s.

27. Loi S, Michiels S, Salgado R, Sirtaine N, Jose V, Fumagalli D, KellokumpuLehtinen PL et al. Tumor infiltrating lymphocytes is prognostic and predictive for trastuzumab benefit in early breast cancer: results from the FinHER trial. Ann Oncol. 2014;7.

28. Bianchini G, Gianni L. The immune system and response to HER2-targeted treatment in breast cancer. Lancet Oncol. 2014;15(2):e58-68. doi:10.1016/ 1470-2045(13)70477-7.

29. Topalian SL, Drake CG, Pardoll DM. Targeting the PD-1/B7-H1(PD-L1) pathway to activate anti-tumor immunity. Curr Opin Immunol. 2012;24(2):207-12. doi:10.1016/j.coi.2011.12.009.

30. Shevach EM. Mechanisms of foxp $3^{+} \mathrm{T}$ regulatory cell-mediated suppression. Immunity. 2009;30(5):636-45. doi:10.1016/j.immuni.2009.04.010.

31. Pagès FKA, Mlecnik B, Asslaber M, Tosolini M, Bindea G, Lagorce C, et al. In situ cytotoxic and memory $\mathrm{T}$ cells predict outcome in patients with earlystage colorectal cancer. J Clin Oncol. 2009;27(35):5944-51.

32. Dieci MV, Criscitiello C, Goubar A, Viale G, Conte P, Guarneri V, et al Prognostic value of tumor-infiltrating lymphocytes on residual disease after primary chemotherapy for triple-negative breast cancer: a retrospective multicenter study. Ann Oncol. 2014;25(3):611-8. doi:10.1093/annonc/mdt556.

33. Ono M, Tsuda H, Shimizu C, Yamamoto S, Shibata T, Yamamoto H, et al Tumor-infiltrating lymphocytes are correlated with response to neoadjuvant chemotherapy in triple-negative breast cancer. Breast Cancer Res Treat. 2012;132(3):793-805. doi:10.1007/s10549-011-1554-7.

34. Muenst S, Soysal SD, Gao F, Obermann EC, Oertli D, Gillanders WE. The presence of programmed death 1 (PD-1)-positive tumor-infiltrating lymphocytes is associated with poor prognosis in human breast cancer. Breast Cancer Res Treat. 2013;139(3):667-76. doi:10.1007/s10549-013-2581-3.

35. Sun S, Fei X, Mao Y, Wang X, Garfield DH, Huang O, et al. PD-1(+) immune cell infiltration inversely correlates with survival of operable breast cancer patients. Cancer Immunol Immunother CII. 2014;63(4):395-406. doi:10.1007/ s00262-014-1519-x.

36. Mahmoud SM, Paish EC, Powe DG, Macmillan RD, Lee AH, Ellis IO, et al. An evaluation of the clinical significance of $\mathrm{FOXP}^{+}$infiltrating cells in human breast cancer. Breast Cancer Res Treat. 2011;127(1):99-108. doi:10.1007/ s10549-010-0987-8.

37. Mahmoud SM, Paish EC, Powe DG, Macmillan RD, Grainge MJ, Lee AH, et al Tumor-infiltrating CD8 ${ }^{+}$lymphocytes predict clinical outcome in breast cancer. J Clin Oncol. 2011;29(15):1949-55. doi:10.1200/JCO.2010.30.5037.

38. Liu S, Lachapelle J, Leung S, Gao D, Foulkes WD, Nielsen TO. CD8 ${ }^{+}$lymphocyte infiltration is an independent favorable prognostic indicator in basallike breast cancer. Breast Cancer Res BCR. 2012;14(2):R48. doi:10.1186/ bcr3148.

39. Rathore AS, Kumar S, Konwar R, Srivastava AN, Makker A, Goel MM Presence of $\mathrm{CD}^{+}$tumor infiltrating lymphocytes is significantly associated with good prognosis in infiltrating ductal carcinoma of breast. Indian $\mathrm{J}$ Cancer. 2013;50(3):239-44. doi:10.4103/0019-509X.118744.

40. Adams S, Gray RJ, Demaria S, Goldstein L, Perez EA, Shulman LN, et al Prognostic value of tumor-infiltrating lymphocytes in triple-negative breas cancers from two phase III randomized adjuvant breast cancer trials: ECOG 2197 and ECOG 1199. J Clin Oncol. 2014;. doi:10.1200/JCO.2013.55.0491.

41. Yamaguchi R, Tanaka M, Yano A, Tse GM, Yamaguchi M, Koura K, et al. Tumor-infiltrating lymphocytes are important pathologic predictors for neoadjuvant chemotherapy in patients with breast cancer. Hum Pathol. 2012;43(10):1688-94. doi:10.1016/j.humpath.2011.12.013.

42. Lee HJ, Seo JY, Ahn JH, Ahn SH, Gong G. Tumor-associated lymphocytes predict response to neoadjuvant chemotherapy in breast cancer patients. J Breast Cancer. 2013;16(1):32-9. doi:10.4048/jbc.2013.16.1.32.

43. West NR, Milne K, Truong PT, Macpherson N, Nelson BH, Watson PH. Tumorinfiltrating lymphocytes predict response to anthracycline-based chemotherapy in estrogen receptor-negative breast cancer. Breast Cancer Res BCR. 2011;13(6):R126. doi:10.1186/bcr3072.

44. Loi S, Sirtaine N, Piette F, Salgado R, Viale G, Van Eenoo F, et al. Prognostic and predictive value of tumor-infiltrating lymphocytes in a phase III randomized adjuvant breast cancer trial in node-positive breast cancer comparing the addition of docetaxel to doxorubicin with doxorubicin-based chemotherapy: BIG 02-98. J Clin Oncol. 2013;31(7):860-7. doi:10.1200/JCO.2011.41.0902.

45. Matkowski RGI, Halon A, Lacko A, Szewczyk K, Staszek U, Pudelko M, et al. The prognostic role of tumor-infiltrating CD4 and CD8 T lymphocytes in breast cancer. Anticancer Res. 2009;29(7):2445-51. 